\title{
DOI: https://doi.org/10.46296/yc.v4i7edesp.0082
}

\section{RIESGO PSICOSOCIAL EN TRABAJADORES BANCARIOS DE UNA COOPERATIVA EN LA CIUDAD DE CHONE, ECUADOR}

\section{PSYCHOSOCIAL RISK IN BANK WORKERS OF A COOPERATIVE IN THE CITY OF CHONE, ECUADOR}

\author{
Vélez-Villalva Yenni Mariuxi ${ }^{1 *}$; Salvador-Moreno Janeth Elizabeth² \\ ${ }^{1}$ Maestrante en Administración de Empresas, mención: Gerencia de calidad y productividad, \\ Pontificia Universidad Católica del Ecuador - sede Manabí, PUCESM. Portoviejo, Ecuador. \\ Orcid: https://orcid.org/0000-0002-2021-7499
}

${ }^{2}$ Pontificia Universidad Católica del Ecuador - Sede Manabí, PUCESM. Portoviejo, Ecuador.

Orcid: https://orcid.org/0000-0003-4252-9271

*Correo: yvelez8697@pucem.edu.ec

\begin{abstract}
Resumen
Las condiciones y ambientes laborales se modifican permanentemente, denotándose cambios que pueden inducir a la prevalencia de factores de riesgo asociados con diferentes factores del entorno laboral. En este sentido, los riesgos psicosociales constituyen una problemática en constante crecimiento y las instituciones bancarias no se encuentran exentas de esta tendencia. Por lo tanto, el objetivo de la presente investigación es analizar los riesgos psicosociales a los que se exponen los trabajadores bancarios de una cooperativa en la ciudad de Chone, Manabí Ecuador y así determinar cuál es el área con mayor nivel de riesgo psicosocial e identificar cuáles son las principales causas de estrés en los trabajadores. Se utilizó un instrumento investigativo categorizado en 9 ítems, según la metodología planteada en la herramienta FPSICO 4.0 propuesta por el INSST-España, con una muestra de 30 participantes. Se concluye que, en las políticas de regulación y control de riesgos laborales se exponen las medidas que deben adoptarse por las instituciones según la naturaleza de sus actividades, no obstante, un sector importante de las organizaciones no las ejecuta y por lo tanto, se presenta una percepción de los trabajadores que evidencia falencias en el cuidado y prevención de las condiciones laborales.
\end{abstract}

Palabras clave: Riesgos laborales, psicosocial, factores, trabajadores, cooperativas.

\begin{abstract}
Work conditions and environments are permanently modified, denoting changes that may induce the prevalence of risk factors associated with different factors in the work environment. In this sense, psychosocial risks constitute a constantly growing problem and banking institutions are not exempt from this trend. Therefore, the objective of this research is to analyze the psychosocial risks to which the bank workers of a cooperative in the city of Chone, Manabí Ecuador are exposed and thus determine which is the area with the highest level of psychosocial risk and identify which ones they are the main causes of stress in workers. An investigative instrument categorized into 9 items was used, according to the methodology proposed in the FPSICO 4.0 tool proposed by INSST-Spain, with a sample of 30 participants. It is concluded that, although the policies for the regulation and control of occupational risks set out the measures that must be adopted by the institutions according to the nature of their activities, nevertheless, an important sector of the organizations does not execute them and therefore, a perception of workers is presented that shows shortcomings in the care and prevention of working conditions.
\end{abstract}

Keywords: Occupational risks, psychosocial, factors, workers, cooperatives.

Información del manuscrito:

Fecha de recepción: 02 de septiembre de 2020

Fecha de aceptación: 28 de septiembre de 2020

Fecha de publicación: 16 de noviembre de 2020 


\section{Introducción}

Los diversos y constantes cambios de la economía y de las diferentes organizaciones empresariales introducen variantes relevantes en el mundo laboral (Muñoz-Rojas et al., 2018). De acuerdo con la publicación del Banco Estudiantil (2018) el sistema financiero ecuatoriano se encuentra compuesto por instituciones privadas (bancos, sociedades, cooperativas y mutualistas) y organismos públicos; con el financiamiento obtenido a través del sistema de finanzas, las empresas 0 instituciones gubernamentales realizan inversión productiva, lo que genera un mayor número de empleos; en consecuencia, se puede alcanzar un mayor desarrollo y crecimiento económico.

En Ecuador, según la Superintendencia de Bancos (2018), los bancos constituyen el mayor y más importante participante del mercado con más del $90 \%$ de las operaciones del total del sistema (ICEX, 2018).

Por su parte, el artículo 1 del Instrumento Andino de Seguridad y Salud en el Trabajo define al riesgo laboral como la probabilidad de que la exposición a un factor ambiental peligroso en el trabajo cause enfermedad o lesión. La salud y el bienestar son objetivos primordiales, la conservación de una condición saludable es también una importante aspiración de cualquier ambiente laboral. Las condiciones de trabajo aceptables constituyen un elemento clave para la consecución de tales objetivos (IESS, 2000).

En el trabajo, existen factores de riesgo que son susceptibles de ocasionar trastornos que, sin ser de naturaleza física pueden ocasionar daños a la salud del trabajador. Pueden materializarse en dolencias - lesiones de tipo somático o psicosomático y son perjudiciales a la salud psíquica y social, según el Instituto Sindical de Trabajo, Ambiente y Salud (ISTAS, s.f.).

El Departamento de Asistencia Técnica para la Prevención de Riesgos Laborales de la Unión General de Trabajadores de Andalucía (UGT Andalucía, s.f.), se refiere a riesgo psicosocial como aquellas condiciones presentes en una situación laboral directamente relacionada con la organización del trabajo y la realización de la tarea que se presentan con capacidad 
para afectar el desarrollo del trabajo y la salud del trabajador.

La Organización Mundial de la Salud (OMS, 2019) indica que existen muchos factores del entorno laboral que pueden afectar a la salud mental. En la mayoría de los casos, los riesgos que conllevan se deben a una interacción inadecuada entre el tipo de trabajo, el entorno organizativo y directivo, las aptitudes y competencias del personal. Según las estimaciones de la Organización Mundial de la Salud (OMS, 2019), en el mundo hay 264 millones de personas que padecen depresión, muchas de ellas sufren también síntomas de ansiedad. Se ha estimado que cuestan anualmente a la economía mundial US\$1 billón en pérdidas de productividad.

En la VII Encuesta Nacional de Condiciones de Trabajo realizada por el INSHT (2011), con una muestra de 8.892 trabajadores, se manifiesta que varios indicadores relativos a las exigencias derivadas de factores psicosociales del trabajo como: el nivel de atención exigida en la tarea, con la percepción de tener mucho trabajo y sentirse agobiado, tener que trabajar muy rápido o deber atender varias tareas al mismo tiempo; han empeorado respecto a cuatro años antes.

El Instituto Ecuatoriano de Seguridad Social (IESS, 2000), respecto a los derechos y obligaciones de los trabajadores, indica que: todos los trabajadores tienen derecho a desarrollar sus labores en un ambiente de trabajo adecuado y propicio para el pleno ejercicio de sus facultades físicas y mentales, que garanticen su salud, seguridad y bienestar. Según el capítulo IV. De los derechos y obligaciones de los trabajadores, Art. 18, en todo lugar de trabajo se deberán tomar medidas tendientes a disminuir los riesgos laborales. Estas medidas deberán basarse, para el logro de este objetivo, en directrices sobre sistemas de gestión de la seguridad y salud en el trabajo y su entorno como responsabilidad social y empresarial.

Durante mucho tiempo este tema fue evadido por los empleadores, queriendo minimizar las afectaciones de los diversos ambientes y situaciones a las que están expuestos los colaboradores, sin embargo, el bajo rendimiento de muchos empleados obligó a identificar las causas de la baja 
productividad, esto trajo a la luz la afectación de los empleados por causas de estrés, exposición con los múltiples problemas de los clientes, presión por el cumplimiento de las metas, cargas horarias poco alentadoras y varios factores más como expresa Díaz-Naranjo (2011) en su artículo Estrés laboral y sus factores de riesgo psicosocial.

Para poder determinar el nivel de afectación, es necesario el reconocimiento de qué los factores de Riesgo Psicosocial (RPS) requieren ser evaluados sistemáticamente, lo cual surge en un contexto de transformaciones sociales, políticas y legislativas que acentúan el interés por fomentar la salud en los trabajadores (Charria et al., 2011).

Cabe señalar también que la Cooperativa definida para la presente investigación se encuentra ubicada en Chone, Ecuador; además, cuenta con una estructura organizacional conformada por gerencia, secretaría, auditoría interna, jefes departamentales: riesgo, cumplimiento, marketing, departamento legal, talento humano, financiero (contabilidad y tesorería), captaciones, jefatura de cajas y sus cajeros, negocios (crédito y cobranzas), capacitaciones (atención al usuario) y jefe operativo. Se trata de una institución financiera que atiende hasta 50 solicitudes de créditos por día, con un total de 5 asesores, puesto que la mayor presión con los clientes se registra en el área de créditos. En el área de atención al cliente, atienden 2 personas con un promedio de 30 transacciones diarias cada uno. Entre los principales productos que se ofrecen se encuentran créditos en segmentos: microcréditos, de vivienda y de consumo; entre los servicios: emisión de tarjetas de débito, depósitos a plazo fijo y pagos de servicios básicos. Por su parte, se ha determinado que en estas Cooperativas se realizan dos capacitaciones en el año en las diferentes áreas comerciales, productivas, de gestión y riesgos laborales.

Por lo tanto, la investigación tiene como objetivo analizar los riesgos psicosociales a los que están expuestos los trabajadores bancarios de una cooperativa en la ciudad de Chone, y así determinar cuál es el área con mayor nivel de riesgo psicosocial e identificar cuáles 
son las principales causas de estrés en los trabajadores.

\section{Metodología}

El presente trabajo de investigación es de tipo transversal, dado que su naturaleza documental-cualitativa, se desarrolló bajo una investigación histórica-analítica que busca obtener información de los aspectos centrales, con el objeto de conocer los Riesgos Psicosociales del sistema financiero, las causas que lo provocan y el área más afectada 0 de mayor riesgo. Además de un enfoque cuantitativo por medio de la aplicación del instrumento FPsico 4.0, para ello se determinó una muestra representativa de personas que fueron evaluadas con el fin de obtener resultados confiables en la investigación.

El estudio se delimitó en la ciudad de Chone, Ecuador y es necesario aplicar técnicas e instrumentos que permitieron establecer un contacto con el entorno en el que se está investigando.

El diseño de la investigación que se implementó es de tipo descriptivo, puesto que permitió considerar el fenómeno de estudio y sus componentes, también la asociación de variables que permitieron cuantificar los datos, y conocer la relación existente entre el acceso a microcréditos y el empoderamiento en mujeres emprendedoras. Además, se logró explicar las características del empoderamiento de la mujer, dado que describe tendencias de un grupo poblacional.

La muestra fue tomada en la ciudad de Chone y determinada mediante la unidad de análisis como una cooperativa ubicada en la ciudad de forma aleatoria, para que todas las cooperativas puedan tener la oportunidad de ser elegidas.

El Instituto Nacional de Seguridad y Salud en el trabajo define a FPsico 4.0 como un procedimiento de evaluación psicosocial. Se trata de una aplicación informática que desarrolla el método de evaluación de factores psicosociales diseñado por el Instituto Nacional de Seguridad y Salud en el Trabajo (INSST), para facilitar la identificación y evaluación de los factores de riesgo psicosocial. En este instrumento de investigación se consideran 44 preguntas, algunas con opciones múltiples, de modo que se definen 89 ítems sustentados en 9 factores que se plantean a 
continuación: tiempo de trabajo (TT), autonomía (AU), carga de trabajo (CT), demandas psicológicas (DP), variedad-contenido (VC), participación-supervisión (PS), interés por el trabajadorcompensación (ITC), desempeño de rol (DR), relaciones y apoyo social (RAS).

Esta metodología ha sido propuesta para generar evaluaciones colectivas en aspectos particularmente homogéneos. Además, se menciona que para la aplicación de dicho instrumento, es necesario considerar los siguientes criterios de inclusión: (i) los participantes deberán conocer la intencionalidad del cuestionario; (ii) debe respetarse la privacidad de los participantes, en aras de garantizar la veracidad de las respuestas; (iii) respetar la confidencialidad de los resultados; (iv) disponer de un sistema para aclarar interrogantes de los participantes; (v) evitar la comunicación entre participantes para disminuir las posibilidades de sesgo; (vi) el participante debe ser consciente de que no puede dejar en blanco ningún literal. En cuanto a los criterios de validez y fiabilidad, el procedimiento de baremación (mediante un cuadro gradual para evaluar) permitió interpretar los resultados obtenidos en las escalas de cada ítem. La aplicación informática aporta el resultado baremado, de modo que se establece una escala de colores que permite clasificar a los riesgos en: muy elevado, elevado, moderado y situación adecuada, en función de la combinación de las respuestas que se obtuvieron mediante el método psicrométrico del formulario $y$ generando gráficos con distitnos tonos de color según los 4 niveles mencionados (INSST, 2020).

En cuanto a los participantes de la investigación, se tuvo una muestra de 30 personas, de los cuales 13 son de sexo masculino y 17 de sexo femenino. Asimismo, se analizaron los parámetros planteados en la metodología del test FPsico 4.0 del Instituto Nacional de Seguridad y Salud en el Trabajo (INSST, 2020).

\section{Resultados y discusión}

Mediante la presente investigación se han determinado los resultados que se exponen a continuación. En primer lugar, se exponen los datos sociodemográficos de la población. En su totalidad, se evidenció una participación de 30 personas; de las 
cuales, el $56,66 \%$ se encuentra entre los 25-34 años, el 16,66\% entre 3544 años y $26,67 \%$ entre $45-54$ años de edad. El $56,67 \%$ de los participantes son de sexo femenino y el restante $(43,33 \%)$ de sexo masculino. En lo que respecta al área de trabajo, el $63,33 \%$ de los participantes pertenecen al área administrativa de la entidad financiera y el 36,67\% corresponden al área operativa/servicio. Además, el $26,67 \%$ de los participantes tienen nombramiento y el $73,33 \%$ cuenta con contrato. Por su parte, el $30 \%$ tienen una antigüedad institucional de 1-5 años, el 30\% entre 6-10 años y el restante (40\%) igual o superior a los 11 años. Se demostró también que el $56,67 \%$ tienen una antigüedad en el puesto de trabajo de 1-5 años, el $23,33 \%$ de $6-10$ años y un $20 \%$ tiene una antigüedad en el puesto superior a los 11 años. Finalmente, el $73,33 \%$ tienen una escala salarial que oscila entre $\$ 500-\$ 1500$ y el $26,67 \%$ entre $\$ 1501-\$ 2500$.

\subsection{Valoración de la exposición a los factores de riesgo psicosocial}

La figura 1 muestra la valoración que los participantes han manifestado respecto a la exposición a factores de riesgo psicosocial. Los factores considerados son: tiempo de trabajo (TT), autonomía (AU), carga de trabajo (CT), demandas psicológicas (CP), variedad/contenido (VC), participación/supervisión (PS), interés por el trabajador/compensación (ITC), desempeño de rol (DR), relaciones y apoyo social (RAS). La figura siguiente evidencia que la gran mayoría de los factores están compuestos por respuestas positivas (barras mayoritariamente verdes), no obstante, el factor Participación/Supervisión tan solo refleja un $20 \%$ de situaciones adecuadas, denotándose que el $43,3 \%$ refleja riesgos elevados, el $20 \%$ riesgo moderado y el restante $16,7 \%$ riesgo muy elevado.

Es importante considerar, que en nuestro medio son muy latentes los factores de riesgo psicosocial, dado que como se refleja en la presente investigación, desde la organización financiera no se toman todas las medidas pertinentes para mitigar la totalidad de factores de riesgo, constituyéndose en elementos que pueden afectar a la seguridad y salud de los trabajadores en el corto, mediano y largo plazo. Por su parte, 
a nivel europeo se ha demostrado que el $73,1 \%$ de los establecimientos de la Unión Europea toman medidas preventivas contra el daño físico directo y alrededor del 35,4\% toman medidas para prevenir los riesgos psicosociales. Se identificaron vínculos débiles e inconsistentes entre las políticas y los indicadores de prácticas de sistemas de seguridad y salud ocupacional (Verra et al., 2019). Por lo tanto, la percepción de los trabajadores que se obtuvo en el presente estudio se asocia con las políticas de seguridad y salud, puesto que evidentemente se concentran en la prevención, mientras que el cumplimiento de las prácticas de salud y seguridad es relativamente bajo. Los riesgos psicosociales a menudo se abordan en las políticas y mecanismos de control y regulación público, pero las instituciones no los implementan.

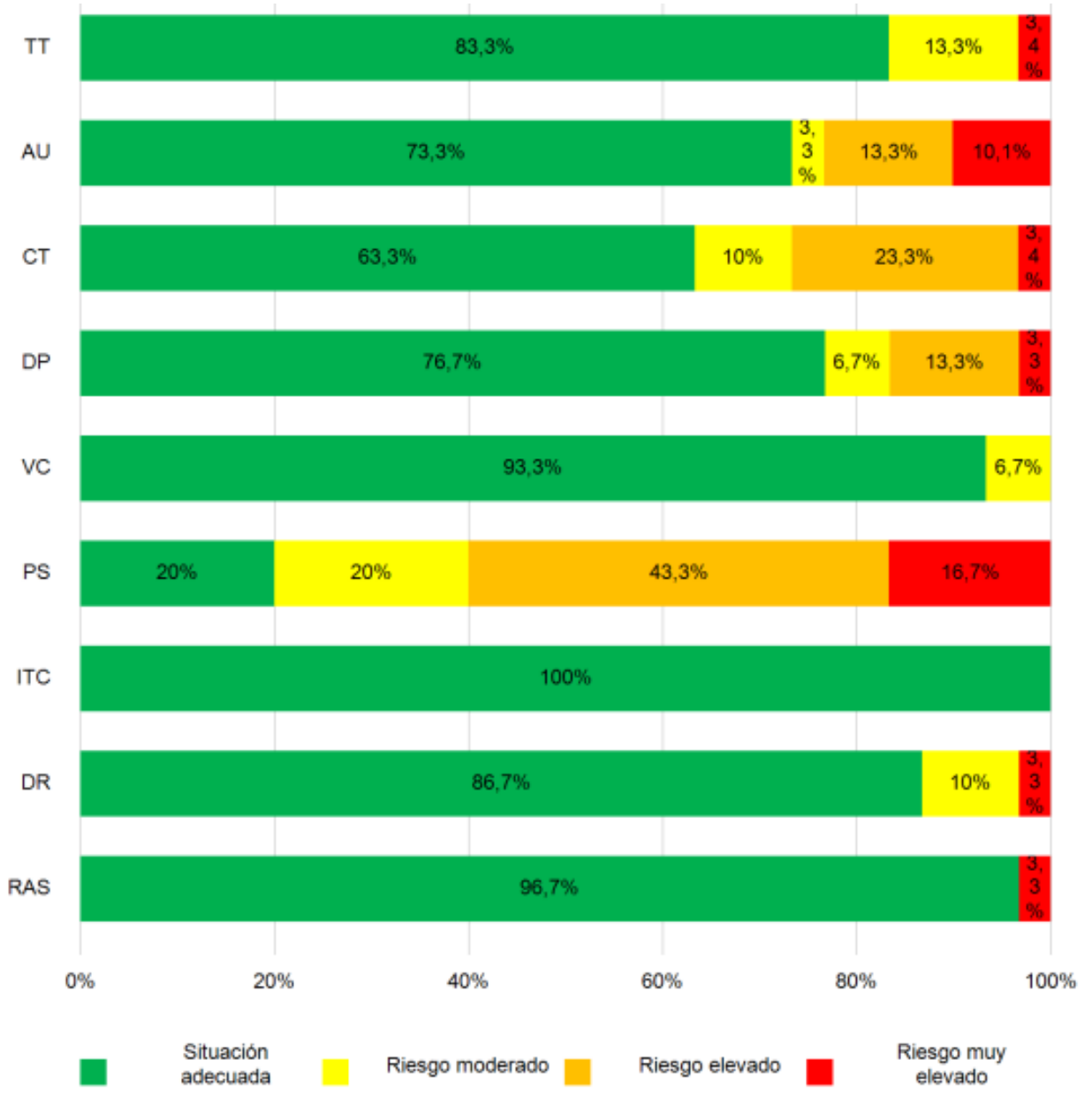

Figura 1. Valoración de la exposición 


\subsubsection{Participación/supervisión}

Es evidente que el nivel de participación depende de varios factores; sin embargo, en el sexo se demuestra que, para los hombres, el $69,2 \%$ solo recibe información y el $30,8 \%$ no tiene ninguna participación. Mientras tanto, en las mujeres, el $23,5 \%$ expresa que se les consulta, el $58,8 \%$ señala que solo reciben información y el restante $17,7 \%$ no tienen ninguna participación. Se observa una tendencia constante tanto en hombres como mujeres en cuanto a la escasa libertad para tomar decisiones y a la permanente dependencia de las acciones y decisiones de los jefes o inmediatos superiores.

El factor de Participación/supervisión ha sido evaluado en función de la relación contractual, denotándose que el $25 \%$ de los trabajadores con nombramiento perciben situaciones adecuadas de trabajo, el $25 \%$ percibe riesgos moderados y el $50 \%$ percibe riesgos elevados; por su parte, el $40,9 \%$ de los colaboradores con contrato perciben riesgos elevados, el 22,7\% perciben riesgos muy elevados, el 18,2\% perciben riesgos moderados y el $18,2 \%$ señalan que laboran en situaciones adecuadas de trabajo (figura 2).

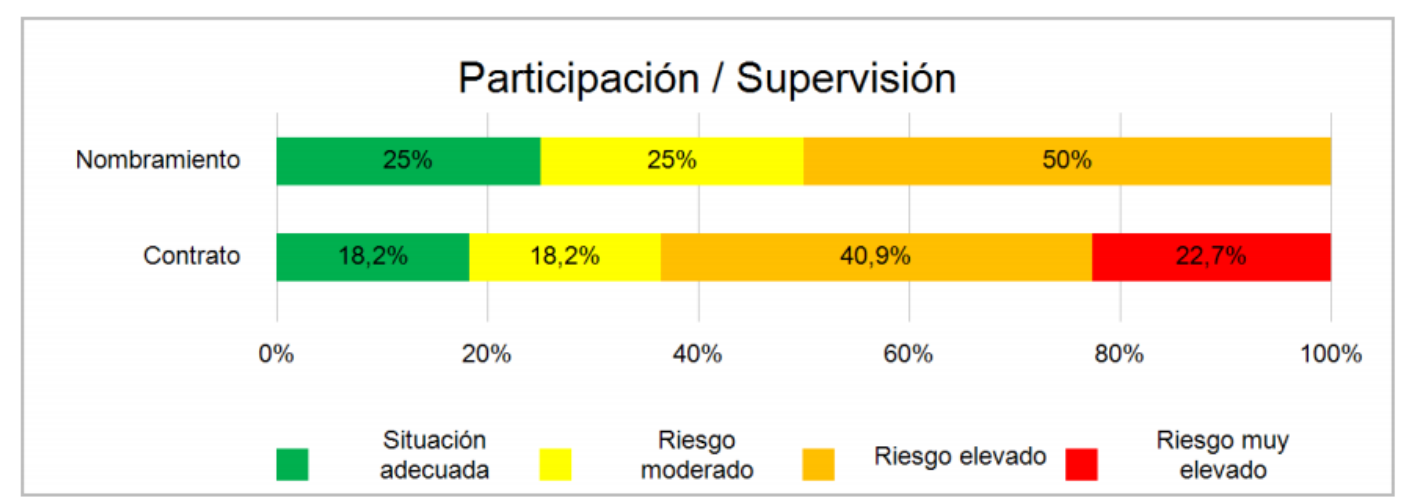

Figura 2. Participación y supervisión en función de la relación contractual

En el aspecto reestructuración o reorganización de departamentos de trabajo se demuestra que en los trabajadores del área administrativa, el $52,6 \%$ se limita a recibir información y en el área 
operativa/servicio,

el $\quad 72,7 \%$ manifiesta que están limitados a recibir información.

\subsubsection{Carga de trabajo}

La figura 1 corrobora que el factor de trabajo (CT) si bien presenta un 63,3\% de situación adecuada, existe la presencia de riesgos psicosociales categorizados de la siguiente forma: $23,3 \%$ de riesgo elevado, $10 \%$ de riesgo moderado y $3,4 \%$ de riesgo muy elevado. Es importante, señalar que en el aspecto cantidad y dificultad de trabajo, en el área administrativa, el $10,5 \%$ de los colaboradores siempre o casi siempre deben trabajar más tiempo del horario habitual, el $21,1 \%$ se exponen a este factor de riesgo psicosocial a menudo, el $47,4 \%$ se exponen a veces y el $21 \%$ nunca o casi nunca. Por su parte, en el área operativa o de servicios, el $27,3 \%$ se exponen a menudo a realizar trabajo adicional al de su horario habitual, el $54,5 \%$ expresa que a veces trabajan más tiempo del horario habitual, mientras que el $18,2 \%$ nunca o casi nunca realizan horas extra, llevan trabajo a casa o trabajan más del horario habitual.

También se reporta que el $46,7 \%$ de los participantes solo recibe información y el 46,6\% no refleja ninguna participación en aspectos como la contratación o incorporación de nuevos empleados, mientras que en la elaboración de las normas de trabajo solo el 6,7\% puede decidir. En lo que respecta a la relación contractual, el $75 \%$ de los colaboradores con nombramiento a menudo deben atender varias tareas al mismo tiempo; sin embargo, el $50 \%$ de los trabajadores con nombramiento a veces debe atender varias tareas al mismo tiempo. Es decir, se demuestra que, en ambos casos, el trabajador realiza varias actividades al mismo tiempo y por tanto, en ocasiones se excede el horario de trabajo.

La figura 3 muestra que la relación contractual tiene un efecto significativo sobre la percepción de los trabajadores respecto a las situaciones laborales y la prevalencia de riesgos psicosociales; de manera que el $25 \%$ de los colaboradores con nombramiento percibe riesgos moderados, al considerar el factor de carga de trabajo; no obstante, en los colaboradores con contrato, el $31,8 \%$ percibe riesgos elevados, el $4,5 \%$ percibe ambientes con riesgos 
moderados y el 4,6\% percibe riesgos muy elevados. En concordancia, De Sio et al. (2018) señalan que la percepción de riesgos psicosociales expone a los trabajadores a desarrollar estrés relacionado con el trabajo. Recientemente, la atención de la investigación científica se ha centrado en un riesgo psicosocial ya identificado como precariedad laboral, que se refiere a la "preocupación general por la relación contractual y la posibilidad de continuar en el trabajo a futuro, que también depende de la percepción del trabajador". Por ende, es importante demostrar el impacto de los riesgos psicosociales sobre la vulnerabilidad del trabajador, puesto que la percepción de los riesgos psicosociales puede verse influida por la inseguridad laboral, en forma de varios factores de riesgo, y aumenta la vulnerabilidad del colaborador al estrés relacionado con el trabajo.

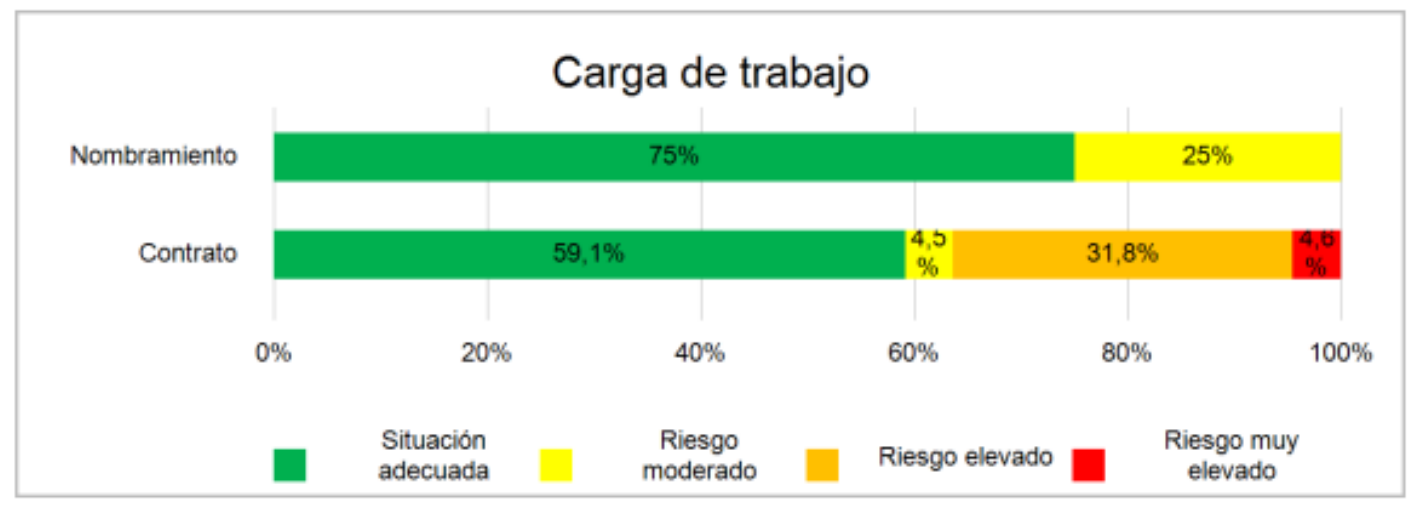

Figura 3. Carga de trabajo en función de la relación contractual

Barahona \& Sarsosa (2008) realizaron una investigación referente a la percepción de los factores de riesgo psicosocial en una institución financiera y determinaron una percepción de riesgo psicosocial de la función de nivel medio; demostrando que los factores de riesgo psicosociales más comunes son: motivación, compromiso, participación y estrés, que tuvieron relación con el rendimiento de los colaboradores, su grado de productividad y agilidad para realizar las tareas designadas. En este sentido, esta investigación se asocia 
parcialmente con estos resultados, puesto que, en este caso particular, de los factores reportados por Barahona \& Sarsosa (2008) tan solo se coincide con la participación y el consecuente estrés que se genera.

Cedeño \& Chávez (2020) realizaron un análisis comparativo entre los resultados de la aplicación de las herramientas ISTAS 21 y FPSICO en una entidad financiera de Portoviejo, Ecuador y determinaron resultados similares a los obtenidos en la presente investigación, puesto que estos autores reportan que el factor Supervisión/Participación de la herramienta FPSICO fue evaluado como riesgo muy alto con el $42 \%$.

Cabe señalar también que, en este mismo enfoque, Weissbrodt et al. (2018) mencionan que las respuestas en el lugar de trabajo a las intervenciones de la inspección del trabajo con respecto a los riesgos psicosociales no se aplican favorablemente. Este estudio tuvo como objetivo evaluar los efectos de las visitas de inspección a nivel de empresa. Se realizó una encuesta en dos grupos de empresas. Se distinguieron dos tipos de medidas de prevención: medidas específicas de gestión del riesgo psicosocial, por un lado, y mejoras más generales de las condiciones laborales, en el otro lado. Los resultados fueron alentadores, ya que las empresas inspeccionadas mejoraron su gestión de salud y seguridad, aumentaron su capacidad en temas psicosociales y demostraron una mayor disposición a actuar. En menor medida, implementaron medidas específicas de gestión del riesgo psicosocial. Sin embargo, las visitas no dieron lugar a mejoras en las puntuaciones de participación/supervisión de los empleados ni a mejoras generales de las condiciones laborales, como la organización del trabajo, los horarios de trabajo o los niveles de personal. Un paso más para las iniciativas regulatorias sería enfatizar en la necesidad de propuestas de prevención basadas en la evaluación y mejora de los diseños, el contenido y la organización de los puestos de trabajo.

Finalmente, Kyaw-Myint et al. (2017) presentaron un enfoque novedoso para identificar niveles de exposición críticos o puntos de referencia de control del trabajo basados en la salud, utilizando el método de dosis 
de referencia. Este método proporciona puntos de referencia para la evaluación de riesgos psicosociales, similares a los puntos de referencia utilizados para otros peligros para la salud ocupacional, como los productos químicos. La depresión es el factor más sensible (compromete la salud) para el control del trabajo en comparación con la ansiedad. Para un exceso de riesgo de depresión del $5 \%$, la dosis de referencia ajustada fue $0,49 \mathrm{y}$ el nivel de exposición crítico, que es el límite de confianza del 95\% unilateral inferior de la MDR ajustada, fue 0,37. Si las pautas del lugar de trabajo se basan en este nivel crítico de exposición, los trabajadores deben tener un mínimo de diez de los 15 aspectos del control del trabajo medidos en este estudio para reducir el riesgo excesivo de depresión.

\section{Conclusiones}

La promoción de estilos de vida saludables y seguros en el trabajo debería complementar los programas de seguridad en el trabajo, puesto que se ha demostrado que condiciones óptimas de trabajo favorecen el desempeño laboral y disminuyen la prevalencia de casos de accidentes o incidentes laborales que pueden afectar económicamente a la institución.

En las instituciones financieras deben promoverse prácticas y políticas que se constituyan en medidas preventivas de riesgos psicosociales, puesto que según el presente estudio se ha determinado la prevalencia de factores de riesgo que en el mediano y largo plazo inciden en la salud y seguridad de los trabajadores. Cabe señalar que, si bien en las políticas de regulación y control de riesgos laborales se exponen las medidas que deben adoptarse por las instituciones según la naturaleza de sus actividades, no obstante, un sector importante de las organizaciones no las ejecuta y por lo tanto, se presenta una percepción de los trabajadores que evidencia falencias en el cuidado y prevención de las condiciones laborales. Los métodos actuales de evaluación de riesgos se encuentran desactualizados y a menudo carecen de indicadores psicosociales. La promoción de la salud en el trabajo es poco común en las políticas y la práctica, y su interpretación sigue siendo netamente de carácter preventivo. 


\section{Bibliografía}

Banco Estudiantil. (2018). Sistema

Financiero del Ecuador.

Quito, Ecuador: BE.

Disponible en:

https://bancoestudiantil.com

/sistema-financiero-del-

ecuador/

Barahona, J. \& Sarsosa, K. (2008).

Percepción de los factores de riesgo psicosocial en una entidad financiera fusionada. Pensamiento Psicológico, 4(11), 183-198.

Cedeño, A. \& Chávez, R. (2020). Análisis comparativo de las herramientas ISTAS $21 \mathrm{y}$ FPSICO en personal del sector financiero. Revista San Gregorio, 39, 143-161. http://dx.doi.org/10.36097/rs an.v1i39.1402

Charria, V. H., Sarsosa, K. V., \& Arenas, F. (2011). Factores de riesgo psicosocial laboral: métodos e instrumentos de evaluación. Revista Facultad Nacional de Salud Pública, 29(4), 380-391.

De Sio, S., Cedrone, F., Trovato Battagliola, E., Buomprisco,
G., Perri, R., \& Greco, E. (2018). The perception of psychosocial risks and workrelated stress in relation to job insecurity and gender differences: a crosssectional study. BioMed Research International, 2018.

https://doi.org/10.1155/2018 17649085

Díaz-Naranjo, D. (2011). Estrés laboral y sus factores de riesgo psicosocial. Revista CES Salud Pública, 2(1), 8084. Disponible en: https://dialnet.unirioja.es/ser vlet/articulo?codigo $=367722$ 9

ICEX. (2018). Sector Financiero en Ecuador. España, Exportación e Inversiones. Disponible en: file://C:/Users/Ana/Downloa ds/DOC2018792917.pdf

IESS. (2000). Instrumento Andino de Seguridad y Salud en el Trabajo. Instituto Ecuatoriano de Seguridad Social. Quito, Ecuador: IESS. Disponible en: https://oiss.org/wp- 
content/uploads/2018/12/de

cision584.pdf

INSST. (2011). VII Encuesta Nacional de Condiciones de Trabajo, 2011. Instituto Nacional de Seguridad y Salud en el Trabajo. Ministerio de Trabajo y Economía Social. Madrid, España: INSST. Disponible en:

https://www.insst.es/docume ntacion/catalogo-depublicaciones/vii-encuestanacional-de-condiciones-detrabajo-2011

INSST. (2020). FPsico 4.0. Instituto Nacional de Seguridad y Salud en el Trabajo. Ministerio de Trabajo, Migraciones y Seguridad Social. Madrid, España: INSST.

ISTAS. (s. f.). Salud laboral. Instituto Sindical de Trabajo, Ambiente y Salud. Madrid, España: ISTAS. Disponible en: https://istas.net/saludlaboral

Kyaw-Myint, S. M., Strazdins, L., Clements, M., Butterworth, P., \& Gallagher, L. (2017). A method of identifying healthbased benchmarks for psychosocial risks at work: A tool for risk assessment. Safety science, 93, 143-151. https://doi.org/10.1016/j.ssci .2016.11.016

Muñoz-Rojas, D., Orellano, N., \& Hernández-Palma, $\mathrm{H}$. (2018). Riesgo psicosocial: tendencias y nuevas orientaciones laborales. Psicogente, 21(40), 532544.

https://doi.org/10.17081/psic o.21.40.3090

OMS. (2019). Salud mental en el lugar de trabajo. Organización Mundial de la Salud. Ginebra, Suiza: OMS. Disponible en: https://www.who.int/mental_ health/in_the_workplace/es/

UGT-Andalucía. (s.f.). Unión General de Trabajadores de Andalucía. Departamento de Asistencia Técnica para la Prevención de Riesgos Laborales. Andalucía, España. Disponible en: http://www.ugtandalucia.com/web/10157/n 
oticias/-

/asset_publisher/gJ7n/conte

nt/departamento-de-

asistencia-tecnica-para-la-

prevencion-de-riesgos-

laborales

Verra, S. E., Benzerga, A., Jiao, B., \& Ruggeri, K. (2019). Health promotion at work: $\mathrm{A}$ comparison of policy and practice across Europe.

Safety and Health at Work, 10(1),

21-29. https://doi.org/10.1016/j.sha w.2018.07.003

Weissbrodt, R., Arial, M., Graf, M., Iff, S., \& Giauque, D. (2018). Preventing psychosocial risks at work: An evaluation study of labour inspectorate interventions. Safety science, 110, 355-362. https://doi.org/10.1016/j.ssci .2018 .08 .024 